\title{
School functioning in 8- to 18-year-old children born after in vitro fertilization
}

\author{
Karin Wagenaar • Manon Ceelen • \\ Mirjam M. van Weissenbruch • Dirk L. Knol • \\ Henriette A. Delemarre-van de Waal • Jaap Huisman
}

Received: 5 November 2007 / Accepted: 17 January 2008/Published online: 13 February 2008

(C) The Author(s) 2008

\begin{abstract}
The aim of this study was to examine the school functioning of 8- to 18-year-old children born after in vitro fertilization (IVF). We compared 233 children born after IVF to 233 matched control children born spontaneously from parents with fertility problems on measures of education level, general cognitive ability, school performance (need for extra help, repeating a grade, special education), and rates of learning and developmental disorders. No differences were found between IVF and control children on these measures of school functioning. More than $60 \%$ of adolescents at secondary school attended high academic levels (with access to high school or university). We conclude that children and adolescents born after IVF show good academic achievement and general cognitive ability. They do not experience any more educational limitations than the naturally conceived children and adolescents of the control group. The tendency of
\end{abstract}

\section{K. Wagenaar $(\bowtie) \cdot J$. Huisman}

Department of Medical Psychology and Institute for Clinical and Experimental Neurosciences, VU University Medical Center, P.O. Box 7057, 1007 MB Amsterdam, The Netherlands

e-mail: k.wagenaar@vumc.nl

M. Ceelen · M. M. van Weissenbruch

Department of Paediatrics and Institute for Clinical and Experimental Neurosciences, VU University Medical Center, Amsterdam, The Netherlands

\section{L. Knol}

Department of Clinical Epidemiology and Biostatistics, VU University Medical Center,

Amsterdam, The Netherlands

H. A. Delemarre-van de Waal

Department of Pediatric Endocrinology and Institute for Clinical and Experimental Neurosciences, VU University Medical Center, Amsterdam, The Netherlands reassuring school functioning already found in younger IVF children has been shown to continue at secondary school age.

Keywords In vitro fertilization . Children and adolescents . School functioning $\cdot$ General cognitive ability . Learning disabilities

\section{Abbreviations \\ IVF in vitro fertilization}

\section{Introduction}

The first birth after in vitro fertilization (IVF) was reported in 1978 [6]. Since then, the numbers of newborns conceived by this technology have been growing rapidly and, today, IVF is part of the modern management of infertility worldwide. Approximately $1-3 \%$ of the children in developed countries have been born after IVF conception and a considerable number of these children meanwhile have reached adolescence or young adulthood.

Shortly after the first IVF births, clinicians and researchers became aware of possible increased physical and psychological risk associated with IVF, which resulted in the evaluation of the children born from it. From the psychological point of view, mental and psychomotor development in the early years was an important focus. Although IVF pregnancies appear to be associated with an increased risk of multiple pregnancy, preterm delivery, low birth weight, cesarean sections, and transfer to a neonatal intensive care unit $[9,13]$, studies on mental and psychomotor development, in general, did not show any differences between IVF and naturally conceived children in the first 3 years of life 
$[2,7,8,10,14,16,21-25]$. Developmental and neurological problems that were found at this young age were mainly related to prematurity, low birth weight, or multiple births $[2,3,15]$. Also, intellectual development around the age of 5 years was investigated and found not to be different in children conceived by IVF compared to naturally conceived children $[4,11,19,20]$.

However, from an age of about 6 years, when school functioning becomes more important and cognitive demands increase, only a limited number of studies have evaluated the school performance of IVF children [12, 17, 18]. Although the data from these three studies indicate normal intellectual and school functioning, it can be questioned as to whether one may be conclusive yet on the overall educational outcome of IVF children. No control groups were used and the children were a maximum of 13 years old $[17,18]$, or the study was conducted on a relatively small number of children [12]. Most importantly, no single study has described IVF children's school functioning at secondary school yet.

At the VU University Medical Center (VUmc) in the Netherlands, IVF has been conducted since the early 1980 , with the first birth occurring in 1986. Since a considerable group of these IVF children have now reached (pre)pubertal years, we performed a large study on the growth, health, and psychological functioning of IVF children born between 1986 and 1995. In this study, IVF children were matched and compared to children born spontaneously from parents with fertility problems. The selection of such a control group gives us the opportunity to equalize important parental differences (such as age of the mothers at birth, desirability of and involvement with the child, and the educational level of the parents) and evaluate the role of IVF as such on the child's development. Considering the mentioned literature on academic performance, the aim of the study described here was to evaluate the school functioning of IVF children and adolescents who are at the end of primary school and at secondary school. This paper describes the education level, general cognitive ability, school performance (need for extra help, repeating a grade, special education), and rates of learning or developmental disorders in our cohort of IVF children aged 8 to 18 years, compared to that of the matched control group.

\section{Materials and methods}

Population and participants

The data described in this paper were derived from a large study performed between March 2003 and March 2006 on the growth, health, and psychological functioning of IVF children born between 1986 and 1995 (see for more details of the study population Ceelen et al. [5]). Families with a singleton child conceived by IVF conception in the VUmc, Amsterdam, the Netherlands, were invited by mail to participate in the study. Twins and children born from other reproductive techniques, such as intracytoplasmic sperm injection (ICSI), were excluded. Of the 354 invited IVF families, 12 children were not reachable because they moved or did not respond. Of the 342 families who could be traced, 96 parents and/or children themselves refused to participate, whereas 246 agreed to take part in the study (representing a response rate of 69\%).

A control group of children spontaneously born between 1986 and 1995 from parents who previously visited the Department of Gynecology of the VUmc with fertility problems was used. Children were selected and matched one-to-one on sex and age ( \pm 3 months) with the IVF children. Initially, 454 control families were invited, of which, 31 families were not reachable and three children were deceased. Of the 420 control families who could be traced, 233 children and their parents wanted to participate in the study (response rate 51\%). This means that for 13 IVF children, no matched control child was found. These 13 IVF children were excluded from the analysis and 233 matched couples were included.

Families who refused to participate in the study received a questionnaire regarding growth, health, and education level of both parents and the child, and other child characteristics. The participating children were significantly younger than non-participants $(12.2 \pm 2.6$ years versus $12.7 \pm 2.4$ years, $p<0.01)$ and mothers of participating children more often had a high education level $(37 \%$ versus $24 \%$ in mothers of nonparticipating children, $p<0.01$ ). Differences were similar for the IVF and control populations.

\section{Procedure and measurements}

The study protocol was approved by the Medical Ethics Committee of the VUmc and the Central Committee of Human Research (CCMO) in The Hague. Parents, and children from 12 years of age, signed informed consent forms prior to participation.

Children who participated in the study were seen at the VUmc for evaluation of their physical and developmental history and present growth, health, and psychological functioning. Shortly before the hospital visit, parents were sent a questionnaire to fill in at home, to make an inventory of their fertility problems and treatment, the course of the pregnancy, and socio-demographic characteristics. For the current paper, the following data were used.

Perinatal and socio-demographic data With respect to the pregnancy, the gestational age and birth weight of the 
children were inquired. Gestational age was obtained by parents' self-report and the birth weight data were obtained from the hospitals' birth certificates (49\%), outpatient clinic reports $(37 \%)$, or self-reporting by the parents (14\%). From the parents, the age of the mother at delivery and the current age of the parents were calculated by using the child's and parents' birth dates and the date of the hospital visit for participation in the study. Parental education level was scored as low (elementary school or lower level of secondary school and vocational training), middle (medium and higher level of secondary school or medium level of vocational training), or high (university or higher level of vocational training), and was evaluated in three ways: educational level of the mother, educational level of the father, and the highest educational level of one of either parent. Furthermore, it was registered whether the mother was a first-time mother (primipara) or not.

Education level of the child In general, data were obtained about the current education level of the child according to the levels of the Dutch school system. Subsequently, secondary school levels were grouped into low (lower level of secondary school), middle (medium level of secondary school), or high (higher level of secondary school, e.g., school levels with access to high school or university).

General cognitive ability To screen whether there are differences in the general cognitive ability between the groups, the results of a national test of educational achievement (CITO) administered around the age of 12 in the last year of primary school were used. From the children in the study group who took the CITO test, permission was sought to use their score. In the Netherlands, this test is almost generally used to determine high school entrance level. The CITO scores correlate with IQ measures of 0.63 at 12 years of age [1].

School performance and learning and developmental disorders School performance was evaluated by the rate of children who needed extra lessons, repeated a grade, or who had attended special education during their school career so far. Moreover, the existence of learning or developmental disorders diagnosis, such as dyslexia, attention deficit hyperactivity disorder (ADHD), or autism, as reported by the parents, was registered.

\section{Statistical analysis}

The Statistical Package for Social Sciences (SPSS) Windows version 12.0 and Stata 8.0, a program for statistical data analysis, were used for the analyses. Perinatal and socio-demographic data were analyzed by SPSS using a paired sample $t$-test for continuous data, McNemar test for dichotomous data, and Wilcoxon signed rank test for nondichotomous data. Perinatal and socio-demographic data that differed significantly between the groups were entered into further statistical analyses as covariates. Group comparisons for school performance and learning and developmental disorders were performed with Stata by using a randomeffects logistic regression. Group comparison for CITO scores was done in SPSS using multiple linear regression.

\section{Results}

Perinatal and socio-demographic data

In Table 1, the characteristics of the 233 IVF and 233 ageand sex-matched control children and their parents are summarized. The mean age of the children was 12.20 years $(\mathrm{SD} \pm 2.61)$ in the IVF group and 12.21 years $(\mathrm{SD} \pm 2.59)$ for the control children (the age range and distribution within both groups are presented in Fig. 1). On average, the IVF children had a significantly shorter gestational age than children in the control group (38.91 versus 39.48 weeks, $t$ $(232)=-2.88, p=0.004)$. There was also a significant difference in the proportion of children born prematurely, i.e., $<37$ weeks $(13 \%$ in the IVF versus $6 \%$ in the control group, $\left.\chi^{2}(1, n=233)=5.92, p=0.015\right)$. Birth weight was significantly lower for IVF children than for controls $(3,217.7$ versus 3,427.6 grams, $t(232)=-3.85, p=<0.001)$ and more IVF children had birth weights below 2,500 grams $(11 \%$ in the IVF versus $3.5 \%$ in the control group, $\left.\chi^{2}(1, n=232)=8.26, p=0.004\right)$.

Mothers' age at delivery and mothers' and fathers' age at assessment did not differ significantly between the groups. In the IVF children, the mothers were significantly more often primipara at their birth than the mothers of the children from the control group $(80 \%$ in the IVF versus $46 \%$ in the control group, $\left.\chi^{2}(1, n=466)=46.44, p<0.001\right)$. Mothers $(Z=-2.66, p=0.008)$ and, to a lesser extent, fathers $(Z=-1.96, p=0.05)$ of IVF children more often had low or middle and less often had a high educational level than parents of children from the control group (see Table 1).

The variables on which the groups differed (gestational age, birth weight, parity, and mothers' educational level) were entered into further statistical analyses as covariates.

Education level and general cognitive ability

Table 2 demonstrates that, at the time of evaluation, 53\% of the children in both groups were in primary school, and $44 \%$ of the IVF and control children attended secondary school. About $2.5 \%$ of the children already attended vocational education or university, had left school, or had a job. 
Table 1 Child and parental characteristics of the in vitro fertilization (IVF) and control groups

\begin{tabular}{|c|c|c|c|}
\hline & $\operatorname{IVF}(n=233)$ & Control $(n=233)$ & $p$ \\
\hline \multicolumn{4}{|l|}{ Child } \\
\hline Boys/girls (\%) & $115(49 \%) / 118(51 \%)$ & $115(49 \%) / 118(51 \%)$ & n.s. \\
\hline \multicolumn{4}{|l|}{ Age at assessment (years) } \\
\hline Mean (SD) & $12.20( \pm 2.61)$ & $12.21( \pm 2.59)$ & n.s. \\
\hline Range & $8-18$ & $8-18$ & \\
\hline \multicolumn{4}{|l|}{ Gestational age (weeks) } \\
\hline Mean (SD) & $38.91( \pm 2.48)$ & $39.48( \pm 1.85)$ & 0.004 \\
\hline Range & $27-44$ & $33-43$ & \\
\hline Premature $(\%)^{\mathrm{a}}$ & $30(13 \%)$ & $14(6 \%)$ & 0.015 \\
\hline \multicolumn{4}{|l|}{ Birth weight (grams) } \\
\hline Mean (SD) & $3,217.7( \pm 626.2)$ & $3,427.6( \pm 554.0)$ & $<0.001$ \\
\hline Range & $870-5,000$ & $1,450-5,110$ & \\
\hline Low birth weight $(\%)^{b}$ & $26(11 \%)$ & $8(3.5 \%)$ & 0.004 \\
\hline \multicolumn{4}{|l|}{ Parents } \\
\hline \multicolumn{4}{|l|}{ Mothers' age at birth (years) } \\
\hline Mean (SD) & $34.62( \pm 3.77)$ & $34.46( \pm 4.01)$ & n.s. \\
\hline Range & $24.83-42.15$ & $23.76-43.26$ & \\
\hline \multicolumn{4}{|l|}{ Mothers' age at assessment } \\
\hline Mean (SD) & $46.82( \pm 4.50)$ & $46.66( \pm 4.27)$ & n.s \\
\hline Range & $35.11-58.04$ & $36.15-57.91$ & \\
\hline \multicolumn{4}{|l|}{ Fathers' age at assessment } \\
\hline Mean (SD) & $49.26( \pm 5.51)$ & $49.32( \pm 5,23)$ & n.s. \\
\hline Range & $36.25-71.79$ & $36.92-65.29$ & \\
\hline Primipara (\%) & $186(80 \%)$ & $107(46 \%)$ & $<0.001$ \\
\hline Maternal education level* & $(n=232)$ & $(n=233)$ & 0.008 \\
\hline Low $(\%)$ & $50(21.5 \%)$ & $35(15 \%)$ & \\
\hline Middle (\%) & $106(45.5 \%)$ & $99(42.5 \%)$ & \\
\hline High (\%) & $76(33 \%)$ & $99(42.5 \%)$ & \\
\hline Paternal education level* & $(n=231)$ & $(n=232)$ & 0.050 \\
\hline Low $(\%)$ & $45(19.5 \%)$ & $35(15 \%)$ & \\
\hline Middle (\%) & $103(44.5 \%)$ & $96(41.5 \%)$ & \\
\hline High (\%) & $83(36 \%)$ & $101(43.5 \%)$ & \\
\hline Parental highest education level* & $(n=232)$ & $(n=233)$ & n.s. \\
\hline Low $(\%)$ & $21(9 \%)$ & $19(8 \%)$ & \\
\hline Middle (\%) & $101(43.5 \%)$ & $88(38 \%)$ & \\
\hline High (\%) & $110(47.5 \%)$ & $126(54 \%)$ & \\
\hline
\end{tabular}

Paired $t$-tests were used to compare the group means, dichotomous data were analyzed using McNemar tests (*Wilcoxon signed rank test) n.s. $=$ not significant

${ }^{\text {a }}$ Premature $=$ born $<37$ weeks of gestation

${ }^{\mathrm{b}}$ Low birth weight=birth weight $<2,500$ grams

At secondary school, a majority of both the IVF and control children $(61 \%$ in the IVF and $67 \%$ in the control group, respectively) attended a high school level (with access to high school or university). Compared to the nonparticipants, at secondary school, participants more often had a high school level and less often had a low school level. This was the case in IVF as well as in the control children.

CITO test scores were used to screen the level of general cognitive ability in both groups. Of the total group of 233 IVF and 233 control children, 101 IVF children (43\%) and 92 control children $(40 \%)$ did take a CITO test. Of the remaining children, 107 children in the IVF (46\%) and 112 children in the control groups (48\%) did not have a CITO score available because they had not taken the CITO test already, and 25 IVF children (11\%) and 29 control children $(12 \%)$ undertook another test in their last year to determine their advice for secondary school.

CITO test scores of 74 IVF children (73\%) and 66 control children $(72 \%)$ were really available for analyses. From the remaining children who did take a CITO test, the score was not received, even after repeated requests to the parents (no significant difference in the response rate between IVF and controls). As shown in Table 2, no significant differences were found for CITO scores in the IVF versus the control groups. In the statistical analysis, the 

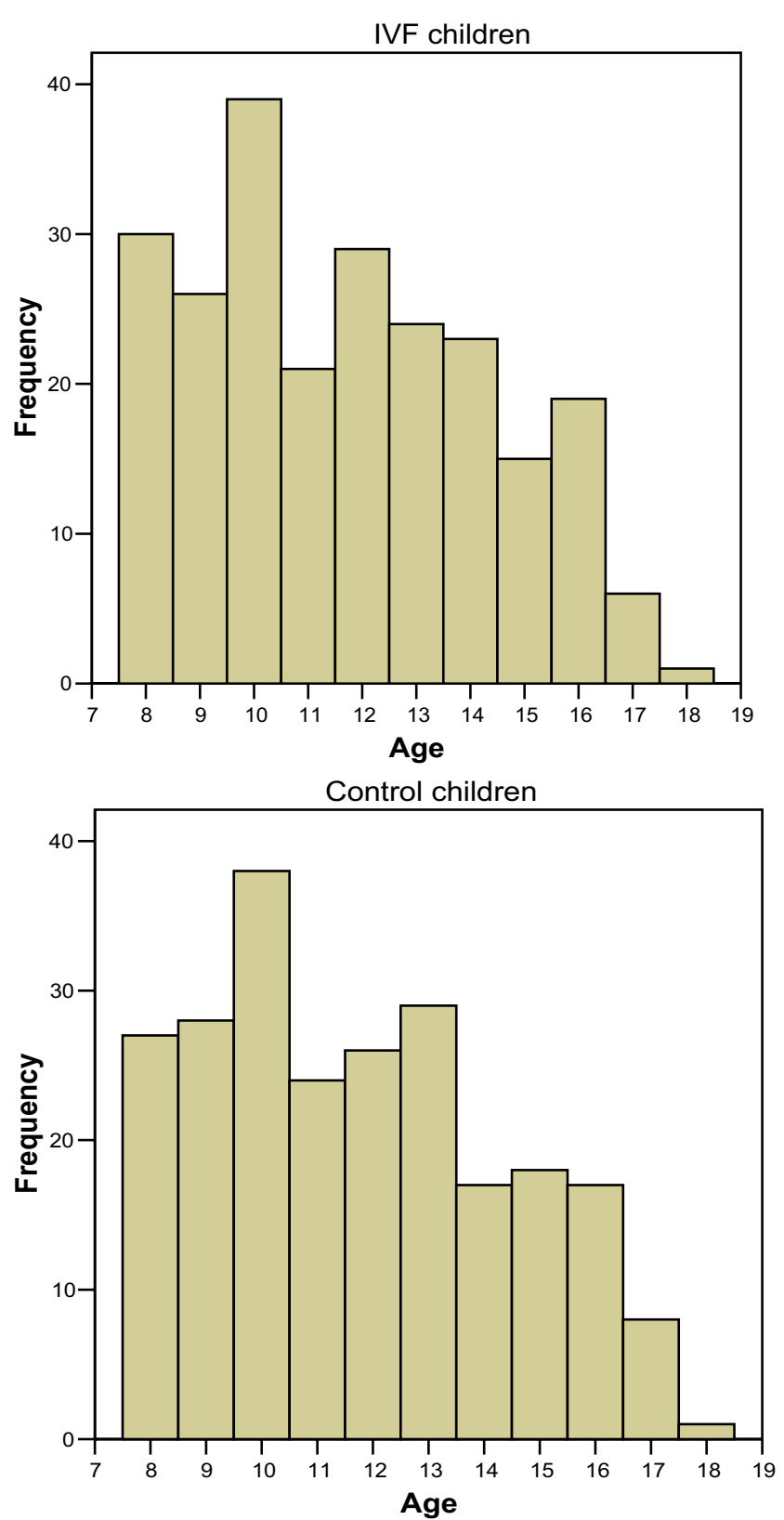

Fig. 1 Age range and distribution within the in vitro fertilization (IVF) and control groups

gestational age, birth weight, parity, and maternal education level were entered as covariates. In both groups, the scores are above the overall Dutch population score (mean score $535)$ and above the score for children from urban districts (mean 538; http://www.cito.nl).

School performance and learning or developmental disorders

As shown in Table 3, no differences were found between the IVF and control groups on the need for extra lessons, repeating a grade, or attending special education. Gestational age, birth weight, parity, and maternal education level were entered in the statistical analysis as covariates. A total of $38 \%$ of the IVF children and $39 \%$ of the control children needed extra lessons and $21 \%$ versus $22 \%$ repeated a grade, most children at nursery and primary school. In both the IVF and control groups, $6 \%$ of the children attended special education (primary and/or secondary school). According to the parents, in $14 \%$ of the IVF children and $18 \%$ of the control children, a learning or developmental disorder has been diagnosed. Also, this difference does not reach statistical significance. Dyslexia is the most common disorder in both groups (about 7\%). Also, 3\% of the IVF and $5 \%$ of the control children have a combination of diagnoses, such as ADHD and an autistic disorder or dyslexia and ADHD. In the IVF group, but not in the control group, motor coordination disorders due to cerebral palsy are present $(n=3)$.

Excluding children with learning or developmental disorders from the analyses did not lead to significant differences between the groups. Rather, a somewhat lower proportion of children in both the IVF and control groups then needed extra lessons, repeated a grade, or attended special education (results not shown in the table).

Table 2 Education level and general cognitive ability in the IVF and control groups

\begin{tabular}{|c|c|c|c|c|}
\hline & $\begin{array}{l}\text { IVF } \\
(n=233) \\
\text { number }(\%)\end{array}$ & $\begin{array}{l}\text { Control } \\
(n=233) \\
\text { number }(\%)\end{array}$ & & $p$ \\
\hline $\begin{array}{r}\text { Primary } \\
\text { school }\end{array}$ & $123(52.8 \%)$ & $124(53.2 \%)$ & & n.s. \\
\hline $\begin{array}{l}\text { Secondary } \\
\text { school }\end{array}$ & $104(44.6 \%)$ & $103(44.2 \%)$ & & n.s. \\
\hline \multicolumn{5}{|l|}{ Level: } \\
\hline Low & $7(6.7 \%)$ & $5(4.9 \%)$ & & n.s. \\
\hline Middle & $34(32.7 \%)$ & $29(28.2 \%)$ & & \\
\hline High & $63(60.6 \%)$ & $69(66.9 \%)$ & & \\
\hline $\begin{array}{l}\text { Vocational } \\
\text { education/ } \\
\text { university }\end{array}$ & $5(2.1 \%)$ & $4(1.7 \%)$ & & n.s. \\
\hline \multirow{3}{*}{$\begin{array}{r}\text { Left school } \\
\text { and/or job }\end{array}$} & $1(0.4 \%)$ & $2(0.9 \%)$ & & n.s. \\
\hline & $\operatorname{IVF}(n=74)$ & Control $(n=66)$ & Adjusted & $p$ \\
\hline & Mean (SD) & Mean (SD) & $\begin{array}{l}\text { difference* } \\
\text { (IVF-control) }\end{array}$ & \\
\hline $\begin{array}{l}\text { CITO test } \\
\text { score }\end{array}$ & $\begin{array}{r}539.43 \\
( \pm 9.5)\end{array}$ & $\begin{array}{r}540.70 \\
( \pm 7.9)\end{array}$ & -1.37 & 0.377 \\
\hline
\end{tabular}

*With covariates parity, maternal education level, gestational age, and birth weight in the univariate linear model

n.s. $=$ not significant 
Table 3 Extra lessons, repeated a grade, special education, and the existence of learning or developmental disorders in the IVF and control groups

\begin{tabular}{|c|c|c|c|c|}
\hline & IVF $(n=233)$ number $(\%)$ & Control $(n=233)$ number $(\%)$ & Adjusted odds ratio* & $p$ \\
\hline Extra lessons & $88(37.8 \%)$ & $91(39.1 \%)$ & 1.02 & 0.927 \\
\hline Repeated a grade & $48(20.6 \%)$ & $52(22.3 \%)$ & 0.94 & 0.800 \\
\hline Nursery school & $17(6.9 \%)$ & $14(6.0 \%)$ & & \\
\hline Primary school & $21(8.5 \%)$ & $20(8,6 \%)$ & & \\
\hline Secondary school & $6(2.4 \%)$ & $5(2.1 \%)$ & & \\
\hline More than once & $1(0.4 \%)$ & $0(0.0 \%)$ & & \\
\hline Repeated a grade followed by special education & $11(4.5 \%)$ & $16(6.9 \%)$ & & \\
\hline Special education & $15(6.4 \%)$ & $16(6.9 \%)$ & 0.90 & 0.791 \\
\hline Learning or developmental disorder & $32(13.7 \%)$ & $42(18.0 \%)$ & 0.73 & 0.310 \\
\hline Dyslexia & $14(6.0 \%)$ & $18(7.7 \%)$ & & \\
\hline Non-verbal learning disorder & $1(0.4 \%)$ & $5(2.1 \%)$ & & \\
\hline Learning disabled & $4(1.7 \%)$ & $5(2.1 \%)$ & & \\
\hline ADHD & $2(0.9 \%)$ & $3(1.3 \%)$ & & \\
\hline Autistic disorder & $1(0.4 \%)$ & $0(0.0 \%)$ & & \\
\hline Motor coordination problems/cerebral palsy & $3(1.3 \%)$ & $0(0.0 \%)$ & & \\
\hline Combination & $7(3.0 \%)$ & $11(4.7 \%)$ & & \\
\hline
\end{tabular}

*With covariates parity, maternal education level, gestational age, and birth weight in the random-effects logistic regression

\section{Discussion}

In this paper, we described the school functioning of a large cohort of IVF singletons born between 1986 and 1995 after conception in the VUmc in the Netherlands. All children, 8-18 years of age, were at the end of primary school or in secondary school, school years on which no conclusive data were yet available. Our findings in IVF children were compared to that of matched control children born spontaneously after a period of subfertility in their parents. The use of this control group gave us the opportunity to equalize as much as possible important parental differences and evaluate the role of IVF as such on the child's development.

We found no indications for educational limitations in IVF children at the end of primary and in secondary school. As many IVF as control children needed extra lessons, repeated a grade, or attended special education. IVF children did not have learning and developmental disorders more often than children from the control group. Also, on general cognitive ability, no differences were found between IVF and control children, and their scores were above the overall Dutch population score and the score for children from urban districts. The distribution of low, middle, or high education level in the children at secondary school did not differ between the groups and was comparable to the distribution found in the parents. More than $60 \%$ of adolescents at secondary school attended a high academic level (with access to high school or university).

Our findings are reassuring and in line with the recent studies in young IVF children [2, 4, 7, 8, 10, 11, 14, 19-25] and at primary school age $[12,17,18]$. Olivennes et al. [17] found, in a follow-up study of 422 IVF children aged 6 to 13 years, according to the French school system, that $92.2 \%$ of the children had at least average school achievement. In 1- to 9-year-old IVF children conceived from cryopreserved embryos, the same authors found comparable results for scholastic performance in the school-age children [18]. Levy-Shiff et al. [12] examined 9- and 10-year-old IVF children in comparison with naturally conceived children recruited from the IVF children's schools and found that intelligence did not differ between the groups. In addition, our findings show also that the tendency of good educational outcome in IVF children continues at secondary school.

However, before drawing any definite conclusions, some aspects have to be considered. With the selected control group in our study, we tried to equalize important parental factors. While most aspects were similar, unfortunately, there were some differences in the education level of the parents. Parents of control participants appeared to be more often higher educated than IVF parents. By using the maternal education level as a covariate, we tried to correct for this difference, as we did also for differences in parity, gestational age, and birth weight. In addition, in our study population, the participants were more highly educated than non-participants. This could have led to an underestimation of the number of children having extra lessons, repeated a grade, attending special education, or having a learning or developmental disorder, and the enhancement of the mean values of the CITO score. Therefore, the precise proportions have to be considered with some caution. Although education level differences were similar in IVF and control (non-)participants, we stress the importance of including lower educated IVF children in future studies. 
Notwithstanding the above, we conclude that the tendency of reassuring school functioning already found in younger IVF children has been shown to continue at secondary school age.

Open Access This article is distributed under the terms of the Creative Commons Attribution Noncommercial License which permits any noncommercial use, distribution, and reproduction in any medium, provided the original author(s) and source are credited.

\section{References}

1. Bartels M, Rietveld MJH, van Baal GCM, Boomsma DI (2002) Heritability of educational achievement in 12-year-olds and the overlap with cognitive ability. Twin Res 5:544-553

2. Brandes JM, Scher A, Itzkovits J, Thaler I, Sarid M, GershoniBaruch R (1992) Growth and development of children conceived by in vitro fertilization. Pediatrics 90:424-429

3. Buitendijk SE (1999) Children after in vitro fertilization. An overview of the literature. Int J Technol Assess Health Care 15:52-65

4. Cederblad M, Friberg B, Ploman F, Sjöberg NO, Stjernqvist K, Zackrisson E (1996) Intelligence and behaviour in children born after in-vitro fertilization treatment. Hum Reprod 11:2052-2057

5. Ceelen M, van Weissenbruch MM, Roos JC, Vermeiden JPW, van Leeuwen FE, Delemarre-van de Waal HA (2007) Body composition in children and adolescents born after in vitro fertilization or spontaneous conception. J Clin Endocrin Metab 92:3417-3423

6. Edwards RG, Steptoe PC (1978) Birth after the reimplantation of a human embryo. Lancet 2:366

7. Gershoni-Baruch R, Scher A, Itskovitz J, Thaler I, Brandes JM (1991) The physical and psychomotor development of children conceived by IVF and exposed to high-frequency vaginal ultrasonography $(6.5 \mathrm{MHz})$ in the first trimester of pregnancy. Ultrasound Obstet Gynecol 1:21-28

8. Gibson FL, Ungerer JA, Leslie GI, Saunders DM, Tennant CC (1998) Development, behaviour and temperament: a prospective study of infants conceived through in-vitro fertilization. Hum Reprod 13:1727-1732

9. Helmerhorst FM, Perquin DAM, Donker D, Keirse MJNC (2004) Perinatal outcome of singletons and twins after assisted conception: a systematic review of controlled studies. BMJ 328:261

10. Koivurova S, Hartikainen AL, Sovio U, Gissler M, Hemminki E, Järvelin MR (2003) Growth, psychomotor development and morbidity up to 3 years of age in children born after IVF. Hum Reprod 18:2328-2336

11. Leslie GI, Gibson FL, McMahon C, Cohen J, Saunders DM, Tennant C (2003) Children conceived using ICSI do not have an increased risk of delayed mental development at 5 years of age. Hum Reprod 18:2067-2072

12. Levy-Shiff R, Vakil E, Dimitrovsky L, Abramovitz M, Shahar N, Har-Even D, Gross S, Lerman M, Levy I, Sirota L, Fish B (1998)
Medical, cognitive, emotional, and behavioral outcomes in school-age children conceived by in-vitro fertilization. J Clin Child Psychol 27:320-329

13. Ludwig AK, Sutcliffe AG, Diedrich K, Ludwig M (2006) Postneonatal health and development of children born after assisted reproduction: a systematic review of controlled studies. Eur J Obstet Gynecol Reprod Biol 127:3-25

14. Morin NC, Wirth FH, Johnson DH, Frank LM, Presburg HJ, Van de Water VL, Chee EM, Mills JL (1989) Congenital malformations and psychosocial development in children conceived by in vitro fertilization. J Pediatr 115:222-227

15. Mushin DN, Barreda-Hanson MC, Spensley JC (1986) In vitro fertilization children: early psychosocial development. J In Vitro Fert Embryo Transf 3:247-252

16. Olivennes F, Fanchin R, Lédée N, Righini C, Kadoch IJ, Frydman R (2002) Perinatal outcome and developmental studies on children born after IVF. Hum Reprod Update 8:117-128

17. Olivennes F, Kerbrat V, Rufat P, Blanchet V, Fanchin R, Frydman R (1997) Follow-up of a cohort of 422 children aged 6 to 13 years conceived by in vitro fertilization. Fertil Steril 67:284-289

18. Olivennes F, Schneider Z, Remy V, Blanchet V, Kerbrat V, Fanchin R, Hazout A, Glissant M, Fernandez H, Dehan M, Frydman R (1996) Perinatal outcome and follow-up of 82 children aged 1-9 years old conceived from cryopreserved embryos. Hum Reprod 11:1565-1568

19. Place I, Englert Y (2003) A prospective longitudinal study of the physical, psychomotor, and intellectual development of singleton children up to 5 years who were conceived by intracytoplasmic sperm injection compared with children conceived spontaneously and by in vitro fertilization. Fertil Steril 80:1388-1397

20. Ponjaert-Kristoffersen I, Bonduelle M, Barnes J, Nekkebroeck J, Loft A, Wennerholm UB, Tarlatzis BC, Peters C, Hagberg BS, Berner A, Sutcliffe AG (2005) International collaborative study of intracytoplasmic sperm injection-conceived, in vitro fertilizationconceived, and naturally conceived 5-year-old child outcomes: cognitive and motor assessments. Pediatrics 115:283-289

21. Raoul-Duval A, Bertrand-Servais M, Frydman R (1993) Comparative prospective study of the psychological development of children born by in vitro fertilization and their mothers. J Psychosom Obstet Gynaecol 14:117-126

22. Raoul-Duval A, Bertrand-Servais M, Letur-Könirsch H, Frydman R (1994) Psychological follow-up of children born after in-vitro fertilization. Hum Reprod 9:1097-1101

23. Ron-El R, Lahat E, Golan A, Lerman M, Bukovsky I, Herman A (1994) Development of children born after ovarian superovulation induced by long-acting gonadotropin-releasing hormone agonist and menotropins, and by in vitro fertilization. J Pediatrics 125:734-737

24. Sutcliffe AG, D'Souza SW, Cadman J, Richards B, McKinlay IA, Lieberman B (1995) Minor congenital anomalies, major congenital malformations and development in children conceived from cryopreserved embryos. Hum Reprod 10:3332-3337

25. Wennerholm UB, Albertsson-Wikland K, Bergh C, Hamberger L, Niklasson A, Nilsson L, Thiringer K, Wennergren M, Wikland M, Borres MP (1998) Postnatal growth and health in children born after cryopreservation as embryos. Lancet 351:1085-1090 